\title{
SOLUTIONS TO THE EINSTEIN-SCALAR FIELD CONSTRAINT EQUATIONS WITH A SMALL TT-TENSOR
}

\author{
ROMAIN GICQUAUD AND THE CANG NGUYEN
}

\begin{abstract}
In this paper, we prove a far-from-CMC result similar to $[15,20,21,29]$ for the conformal Einstein-scalar field constraint equations on compact Riemannian manifolds with positive (modified) Yamabe invariant.
\end{abstract}

\section{CONTENTS}

1. Introduction

2. Preliminaries

2.1. The constraint equations

2.2. The conformal method

3. An implicit function argument

4. An existence result for $\sigma$ and $\pi$ small in $L^{2}$

4.1. The Lichnerowicz equation

4.2. The coupled system

References

\section{INTRODUCTION}

Finding initial data for the Cauchy problem in general relativity is a topical issue. The Einstein equation impose restrictions on the choice of initial data called the constraint equations and finding (physically relevant) solutions to them is the first step in understanding the Cauchy problem in general relativity. We refer the reader to [1] for an extensive description of the constraint equations.

Having in mind the geometric nature of general relativity, initial data for the Cauchy problem are usually given as a triple $(M, \widehat{g}, \widehat{K})$, where $M$ is a manifold (which we will assume closed, i.e. compact without boundary, for simplicity), $\widehat{g}$ is a Riemannian metric on $M$ and $\widehat{K}$ is a symmetric 2-tensor on $M$. The Cauchy problem in general relativity then consists in the following:

Find a space-time, i.e. a Lorentzian manifold $(\mathcal{M}, h)$ solving the Einstein equation

$$
\operatorname{Ric}_{\mu \nu}^{h}-\frac{1}{2} \operatorname{Scal}^{h} h_{\mu \nu}=T_{\mu \nu}
$$

together with an embedding $M \hookrightarrow \mathcal{M}$ such that $M$ becomes a Cauchy surface with induced metric $\widehat{g}$ and second fundamental form $\widehat{K}$.

Here $T$ is the so-called stress-energy tensor of the non-gravitational fields (e.g. matter fields, electromagnetic fields...) one also wants to encompass in the description of the universe.

During the past decades, lots of effort have been dedicated to the study of these equations. However, until fairly recently, the methods could construct only constant mean

Date: $30^{\text {th }}$ July, 2018

Key words and phrases. Einstein constraint equations, non-CMC, conformal method, positive Yamabe invariant, small TT-tensor. 
curvature or almost constant mean curvature initial data. They can be subdivided into two main categories. The first one is the conformal method and its variants which is the method we will use in this article. The second one is the gluing technique introduced by Corvino and Schoen [9].

The conformal method and the closely related conformal thin sandwich method were historically the first methods introduced. The conformal method will be described in Section 2.2. We also refer the reader to the very nice work of Maxwell giving a geometric interpretation of this method [24-26]. The most stricking result of the conformal method is certainly the classification of the set of vacuum $(T \equiv 0)$ constant mean curvature (CMC) solutions to the constraint equations achieved in 1995 by Isenberg in [22] and relies on the solution of the Yamabe problem, see e.g. [23].

The near-CMC case was addressed soon after. We refer the reader to [1] for references.

The far-from-CMC case appears however much more difficult to tackle. It was only in 2008 that Holst, Nagy and Tsogtgerel found a method to construct solutions to the equations of the conformal method with arbitrarily prescribed mean curvature. See [20, 21]. The method was then extended by Maxwell [29] to the vacuum case. Extension to the asymptotically Euclidean case was proven in [11]. Another point of view on this method is given by the first author and Ngo in [15]. While the result of [15] is weaker from a mathematical point of view than the one in [29], the proof appears to be constructive.

The difficulty of the equations of the conformal method in the far-from-CMC case lies in the difficulty to obtain an a priori estimate on the solutions to the equations there is to solve. The method designed by Dahl, Humbert and the first author in [10] can be understood as a criterion for the existence of such an a priori estimate. This method turns out to be particularly efficient with negatively (Ricci) curved metrics, see [16]. See also [12] for the asymptotically cylindrical case.

Simplified proofs of the results in [10, 28] are given by the second author in [30]. A comparative point on view of both methods is given in [15].

Introducing non-gravitational fields in the constraint equations usually leads to not so much more difficult equations to solve for the conformal method because the terms that appear are non critical. There is however an important counterexample to this which is scalar fields. The potential of the field (which also encodes the cosmological constant) can change the sign of one of the dominant terms in the Lichnerowicz equation, see (2.4a). In particular, the method of [10] cannot work any longer and the Lichnerowicz equation may admits multiple solutions, see e.g. [8], [31] and references therein.

The CMC case for the Einstein-scalar field constraint equations was studied in [7] and [18]. The near CMC case was addressed by Premoselli in [32]. We would also like to refer the reader to [5] and [34] for similar results in the non-compact cases.

Based on the ideas developed in [15], [30] and [32], we show that the method of Holst et al. can be extended to the Einstein-scalar field constraint equations.

The outline of the article is as follows. In Section 2 we given the constraint equations with a scalar field and we introduce the conformal method in this context. We then present the extension of the result of [15] in Section 3, see Theorem 3.1. Finally, we address the much more difficult extension of the method of [21] in Section 4, see Theorem 4.1. In the course of the proof, we prove Theorem 4.2 which shows existence of solutions to the Lichnerowicz equation in our context.

The outline of the article is as follows. In Section 2 we give the constraint equations with a scalar field and we introduce the conformal method in this context. We then present the extension of the result of [15] in Section 3, see Theorem 3.1. Finally, we address the much more difficult extension of the method of [21] in Section 4, see Theorem 4.1. In the course of the proof, we prove Theorem 4.2 which shows existence of solutions to the 
Lichnerowicz equation in our context.

Acknowledgements: The authors are grateful to Emmanuel Humbert for his support and his careful proofreading of a preliminary version of this article. The authors also warmly thank Laurent Véron for useful discussions.

\section{Preliminaries}

2.1. The constraint equations. We first recall the derivation of the constraint equations for the Einstein equations with a scalar field $\Psi$, refering to $[1,3,7]$ for further information. The stress-energy tensor of $\Psi$ reads

$$
T_{\mu \nu}=\nabla_{\mu} \Psi \nabla_{\nu} \Psi-\left(\frac{1}{2}|d \Psi|_{h}^{2}+V(\Psi)\right) h_{\mu \nu},
$$

where $h$ denotes the space-time metric and $V$ is the potential of the scalar field. The Einstein-scalar field equations for $h$ and $\phi$ are then

$$
\left\{\begin{aligned}
\operatorname{Ric}_{\mu \nu}^{h}-\frac{1}{2} \operatorname{Scal}^{h} h_{\mu \nu} & =T_{\mu \nu}, \\
\square_{h} \Psi & =V^{\prime}(\Psi) .
\end{aligned}\right.
$$

Let $M$ be a Cauchy surface in the space-time $(\mathcal{M}, h)$ and let $\nu$ denotes its unit futurepointing timelike normal. If $\widehat{g}$ is the metric induced by $h$ on $M$ and if $\widehat{K}(X, Y)=$ $h\left(X, \nabla_{Y} \nu\right)$ is the second fundamental form of $M$, then contracting the first equation in (2.1) with $\nu$ twice gives the Hamiltonian constraint:

$$
\mathrm{Scal}^{\widehat{g}}+\left(\operatorname{tr}_{\widehat{g}} \widehat{K}\right)^{2}-|\widehat{K}|_{\widehat{g}}^{2}=\widehat{\pi}^{2}+|d \widehat{\psi}|_{\widehat{g}}^{2}+2 V(\psi)
$$

where $\widehat{\psi}=\left.\Psi\right|_{M}$ is restriction of $\Psi$ to $M$ and $\widehat{\pi}=\nabla_{\nu} \Psi$ is the time derivative of $\Psi$.

Contracting only once the first equation in (2.1) and restricting the remaining free index to spatial directions (i.e. tangent to $M$ ), we get the momentum constraint:

$$
\operatorname{div}^{\widehat{g}} \widehat{K}-d\left(\operatorname{tr}_{\widehat{g}} \widehat{K}\right)=\widehat{\pi} d \widehat{\psi} .
$$

Hence, solving the constraint equations (2.2)- (2.3) appears to be a necessary condition if one hopes to solve the Einstein equations. Conversly, the celebrated work of ChoquetBruhat [13] and subsequently of Choquet-Bruhat and Geroch [4] ensure that any 5-tuple $(M, \widehat{g}, \widehat{K}, \psi, \widehat{\pi})$ satisfying (2.2)-(2.3) yields a unique solution of the Einstein-scalar field equations (2.1), see also [6]. We refer the reader to [3] and [33] for a comprehensive introduction to the Cauchy problem in general relativity.

2.2. The conformal method. We assume from now on that the manifold $M$ is a given closed manifold of dimension $n$. Counting the degrees of freedom of the 4-tuple $(\widehat{g}, \widehat{K}, \psi, \widehat{\pi})$ and comparing it to the number of equations provided by (2.2) and (2.3), we immediately see that the constraint equations form a (very) underdetermined system. As usual in treating such type of problems, we decompose the variables $(\widehat{g}, \widehat{K}, \widehat{\psi}, \widehat{\pi})$ into given data and unknowns that have to be adjusted to fulfill the constraint equations.

The splitting we will use together with the regularity we will assume are the following (here $p>n$ is given):

- Given (seed) data:

- A (background) metric $g \in W^{2, \frac{p}{2}}$,

- A function $\tau: M \rightarrow \mathbb{R}, \tau \in W^{1, p}$,

- Two functions $\psi \in W^{1, p}$ and $\pi \in L^{p}$,

- A symmetric traceless and divergence free 2-tensor $\sigma \in W^{1, p}$,

- Unknowns:

- A positive function $\phi \in W^{2, \frac{p}{2}}$, 
- A 1-form $W \in W^{2, \frac{p}{2}}$.

From these data, we cook up the initial data as follows:

$$
\widehat{g}=\phi^{N-2} g, \quad \widehat{K}=\frac{\tau}{n} \phi^{N-2} g+\phi^{-2}(\sigma+\mathbb{L} W), \quad \widehat{\psi}=\psi, \quad \widehat{\pi}=\phi^{-N} \pi .
$$

We have used the following notations: $N:=\frac{2 n}{n-2}$ and $\mathbb{L}$ is the conformal Killing operator acting on 1-forms, namely, in coordinates

$$
\mathbb{L} W_{i j}=\nabla_{i} W_{j}+\nabla_{j} W_{i}-\frac{2}{n} \nabla^{k} W_{k} g_{i j},
$$

where $\nabla$ is the Levi-Civita connection associated to the metric $g$.

The constraint equations (2.2)-(2.3) can be rewritten in terms of these new variables:

$$
\begin{aligned}
\frac{4(n-1)}{n-2} \Delta \phi+\mathcal{R}_{\psi} \phi & =\mathcal{B}_{\tau, \psi} \phi^{N-1}+\frac{|\sigma+\mathbb{L} W|^{2}+\pi^{2}}{\phi^{N+1}}, \\
-\frac{1}{2} \mathbb{L}^{*} \mathbb{L} W & =\frac{n-1}{n} \phi^{N} d \tau-\pi d \psi .
\end{aligned}
$$

Equation (2.4a) is usually named the Lichnerowicz equation, while Equation (2.3) is usually refered to as the vector equation. Our convention for the Laplacian is

$$
\Delta \phi:=-g^{i j} \nabla_{i} \nabla_{j} \phi
$$

and the operator appearing in (2.4b) is the vector Laplacian:

$$
-\frac{1}{2} \mathbb{L}^{*} \mathbb{L} W_{j}=\nabla^{i} \mathbb{L} W_{i j}
$$

The functions $\mathcal{R}_{\psi}$ and $\mathcal{B}_{\tau, \psi}$ that appear in the Lichnerowicz equation are given by:

$$
\mathcal{R}_{\psi}=\mathrm{Scal}^{g}-|d \psi|_{g}^{2}, \quad \mathcal{B}_{\tau, \psi}=-\frac{n-1}{n} \tau^{2}+2 V(\psi) .
$$

Compared to the vacuum case (i.e. $\psi, \pi \equiv 0$ ), the coefficient $\mathcal{B}_{\tau, \psi}$ can have arbitrary sign. Also, even if the metric $g$ has positive Yamabe invariant, the generalized conformal Laplacian

$$
L_{g, \psi}: \phi \mapsto \frac{4(n-1)}{n-2} \Delta \phi+\mathcal{R}_{\psi} \phi
$$

is not necessarily a coercive operator, meaning that there may not exists a constant $c>0$ such that

$$
\forall \phi \in W^{1,2}, \int_{M} \phi L_{g, \psi} \phi d \mu^{g} \geqslant c\|\phi\|_{W^{1,2}}^{2} .
$$

This assumption however will turn out to be very important in our analysis and plays a role analog to the assumption that the metric $g$ has positive Yamabe invariant in $[20,21,29]$. Another important assumption we will need is that $(M, g)$ has no non-zero conformal Killing vector field. This assumption is generically true, see [2].

\section{AN IMPLICIT FUNCTION ARGUMENT}

In this section, we show that the method introduced in [15] can be straightforwardly generalized to the system (2.4).

Theorem 3.1. Let $(M, g)$ a closed Riemannian manifold, $\tau \in W^{1, p}, \psi \in W^{1, p}, \widetilde{\pi} \in L^{p}$ and $\widetilde{\sigma} \in L^{p}$ be given. Assume further that the operator $L_{g, \psi}$ defined in (2.5) is coercive and that $(M, g)$ has no non-zero conformal Killing vector field. There exists an $\epsilon_{0}>0$ such that for all $\epsilon \in\left(0, \epsilon_{0}\right)$, the system (2.4) with

$$
\sigma \equiv \epsilon \widetilde{\sigma}, \quad \pi \equiv \epsilon \pi
$$

has a solution $(\phi, W) \in W^{2, \frac{p}{2}} \times W^{2, \frac{p}{2}}$ with $\phi>0$. 
As in the article [15], we divide the proof into several steps:

Step 0. There exists a unique solution $\widetilde{W}_{0} \in W^{2, \frac{p}{2}}$ to

$$
-\frac{1}{2} \mathbb{L}^{*} \mathbb{L} W=-\tilde{\pi} d \psi
$$

Proof. The argument is standard, see e.g. [29, Proposition 5]. Note that $\widetilde{\pi} d \psi \in L^{\frac{p}{2}}$. The operator

$$
-\frac{1}{2} \mathbb{L}^{*} \mathbb{L}: W^{2, \frac{p}{2}} \rightarrow L^{\frac{p}{2}}
$$

is Fredholm with zero index. Its kernel is, by a simple integration by parts argument, the set of conformal Killing vector fields which is reduced to $\{0\}$ by assumption. Hence $-\frac{1}{2} \mathbb{L}^{*} \mathbb{L}$ is an isomorphism.

Step 1. There exists a unique solution $\widetilde{\phi}_{0} \in W^{2, \frac{p}{2}}$ to the following equation:

$$
\frac{4(n-1)}{n-2} \Delta \phi+\mathcal{R}_{\psi} \phi=\frac{\left|\widetilde{\sigma}+\mathbb{L} \widetilde{W}_{0}\right|^{2}+\widetilde{\pi}^{2}}{\phi^{N+1}}
$$

Proof. We set

$$
\widetilde{A}:=\left|\widetilde{\sigma}+\mathbb{L} \widetilde{W}_{0}\right|^{2}+\widetilde{\pi}^{2}
$$

for convenience. Since $\widetilde{W}_{0} \in W^{2, \frac{p}{2}}, \mathbb{L} \widetilde{W}_{0} \in W^{1, \frac{p}{2}} \hookrightarrow L^{p}$. Indeed, from the Sobolev injection, $W^{1, \frac{p}{2}} \hookrightarrow L^{p}$, where

$$
q=\frac{n p}{2 n-p}>p
$$

(here we assumed that $p<2 n$ ). It follows that $\widetilde{A} \in L^{\frac{p}{2}}$. We first prove that there exists a unique positive solution $\bar{\varphi}$ to

$$
\frac{4(n-1)}{n-2} \Delta \phi+\mathcal{R}_{\psi} \phi=\widetilde{A}
$$

We remark that, integrating the righthand side, we get

$$
\begin{aligned}
\int_{M} \widetilde{A} d \mu^{g} & =\int_{M}\left(|\widetilde{\sigma}|^{2}+\tilde{\pi}^{2}\right) d \mu^{g}+\int_{M}|\mathbb{L} W|^{2} d \mu^{g} \\
& \geqslant \int_{M}\left(|\widetilde{\sigma}|^{2}+\tilde{\pi}^{2}\right) d \mu^{g} \\
& >0 .
\end{aligned}
$$

We rely on the Lax-Milgram theorem. Since $L_{g, \psi}$ is coercive, there exists a unique weak solution $\bar{\varphi}$ to (3.3) which is uniquely characterized by

$$
\int_{M}\left(\frac{2(n-1)}{n-2}|d \bar{\varphi}|^{2}+\frac{\mathcal{R}_{\psi}}{2} \bar{\varphi}^{2}-\widetilde{A} \bar{\varphi}\right) d \mu^{g}=\min _{\phi \in W^{1,2}} F(\phi),
$$

where

$$
F(\phi):=\int_{M}\left(\frac{2(n-1)}{n-2}|d \phi|^{2}+\frac{\mathcal{R}_{\psi}}{2} \phi^{2}-\widetilde{A} \phi\right) d \mu^{g} .
$$

Since $\widetilde{A} \geqslant 0$, we have $F(|\phi|) \leqslant F(\phi)$ for any $\phi \in W^{1,2}$. As a consequence $\bar{\varphi}$ being the unique minimizer of $F, \bar{\varphi} \geqslant 0$. By elliptic regularity, we have that $\bar{\varphi} \in W^{2, \frac{p}{2}}$. In particular, $\bar{\varphi}$ is continuous. It can be argued by contradiction that $\bar{\varphi}>0$. Indeed, if the set $\Omega=\{\bar{\varphi}=0\}$ was not empty, it would follows from the Harnack inequality we borrow from [17, Theorem 9.22] applied to $u=\bar{\varphi}$ and $f \equiv 0$ in a ball $B_{R}$ centered at a boundary point of $\Omega$ that $\bar{\varphi} \equiv 0$ on $B_{R}$ which is a contradiction.

Setting $a:=\min _{M} \bar{\varphi}, b:=\max _{M} \bar{\varphi}$, one can readily check that the function

$$
\bar{\varphi}_{+}:=a^{-\frac{N+1}{N}} \bar{\varphi}, \operatorname{resp} \cdot \bar{\varphi}_{-}:=b^{-\frac{N+1}{N}} \bar{\varphi}
$$


is a supersolution (resp. a subsolution) for Equation (3.2). Existence of a solution to (3.2) follows then from the standard sub- and supersolution method, see e.g. [14, Lemma 3.4] or [27]. Uniqueness of $\widetilde{\phi}_{0}$ is also classical, see [10]. However, here we can simply remark that the functional

$$
G(\phi):=\int_{M}\left(\frac{2(n-1)}{n-2}|d \phi|^{2}+\frac{\mathcal{R}_{\psi}}{2} \phi^{2}+\frac{1}{N} \frac{\tilde{A}}{\phi^{N}}\right) d \mu^{g}
$$

is strictly convex on the set of positive $H^{1}$-functions (i.e. so that there exists $\epsilon>0$ such that $\phi \geqslant \epsilon$ a.e.) Its critical points being exactly the solutions to (3.2), we conclude that the solution to (3.2) is unique. This idea will be developed further in Section 4.1.

Step 2. There exists $\epsilon>0$ and $a C^{1}$-map

$$
\begin{array}{ccc}
{[0, \epsilon)} & \rightarrow & W^{2, \frac{p}{2}} \times W^{2, \frac{p}{2}} \\
\lambda & \mapsto & \left(\widetilde{\phi}_{\lambda}, \widetilde{W}_{\lambda}\right)
\end{array}
$$

such that

- $\widetilde{\phi}_{\lambda}$ and $\widetilde{W}_{\lambda}$ solve

$$
\begin{aligned}
\frac{4(n-1)}{n-2} \Delta \widetilde{\phi}_{\lambda}+\mathcal{R}_{\psi} \widetilde{\phi}_{\lambda} & =\lambda^{2} \mathcal{B}_{\tau, \psi} \widetilde{\phi}_{\lambda}^{N-1}+\frac{\left|\widetilde{\sigma}+\mathbb{L} \widetilde{W}_{\lambda}\right|^{2}+\widetilde{\pi}^{2}}{\widetilde{\phi}_{\lambda}^{N+1}} \\
-\frac{1}{2} \mathbb{L}^{*} \mathbb{L} \widetilde{W}_{\lambda} & =\frac{n-1}{n} \lambda \widetilde{\phi}_{\lambda}^{N} d \tau-\widetilde{\pi} d \psi
\end{aligned}
$$

- $\widetilde{\phi}_{\lambda} \rightarrow \widetilde{\phi}_{0}$ and $\widetilde{W}_{\lambda} \rightarrow \widetilde{W}_{0}$ when $\lambda \rightarrow 0$, where $\widetilde{W}_{0}$ and $\widetilde{\phi}_{0}$ are as defined in Steps 0 and 1 .

Note that Equations (3.4) interpolate between the original conformal constraint equations (2.4) when $\lambda=1$ and Equations (3.2)-(3.1) when $\lambda=0$.

Proof. The proof is via the implicit function theorem. Let $\Phi: \mathbb{R} \times W^{2, \frac{p}{2}} \times W^{2, \frac{p}{2}} \rightarrow$ $L^{\frac{p}{2}} \times L^{\frac{p}{2}}$ be the following operator:

$$
\Phi_{\lambda}\left(\begin{array}{c}
\widetilde{\phi} \\
\widetilde{W}
\end{array}\right) \mapsto\left(\begin{array}{c}
\frac{4(n-1)}{n-2} \Delta \widetilde{\phi}+\mathcal{R}_{\psi} \widetilde{\phi}-\lambda^{2} \mathcal{B}_{\tau, \psi} \widetilde{\phi}^{N-1}-\frac{\widetilde{A}}{\phi^{N+1}} \\
-\frac{1}{2} \mathbb{L}^{*} \mathbb{L} \widetilde{W}-\frac{n-1}{n} \lambda \widetilde{\phi}^{N} d \tau+\widetilde{\pi} d \psi
\end{array}\right)
$$

Its differential with respect to the variables $(\widetilde{\phi}, \widetilde{W})$ at $\left(\lambda=0, \widetilde{\phi}_{0}, \widetilde{W}_{0}\right)$ is given by the following block upper triangular matrix:

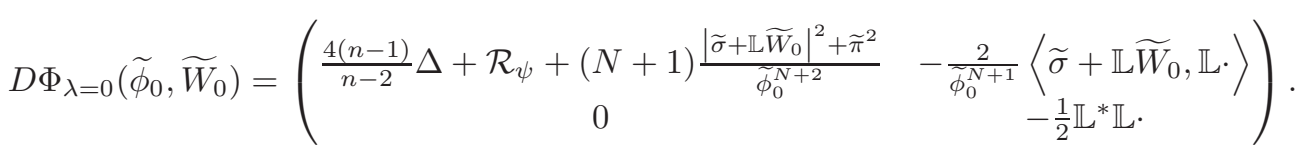

Each diagonal block is Fredholm with zero index and has, by assumption, a trivial kernel. This proves that $D \Phi_{\lambda=0}\left(\widetilde{\phi}_{0}, \widetilde{W}_{0}\right)$ is invertible. The existence of the curve of solutions to (3.4) on some interval $[0, \epsilon)$ is then guaranteed by the implicit function theorem.

The last step is a straightforward calculation.

Step 3. Let $\left(\widetilde{\phi}_{\lambda}, \widetilde{W}_{\lambda}\right)$ be as in Step 2. Setting

$$
\left\{\begin{aligned}
\phi_{\lambda} & :=\lambda^{\frac{2}{N-2}} \widetilde{\phi}_{\lambda}, \\
W_{\lambda} & :=\lambda^{\frac{N+2}{N-2}} \widetilde{W}_{\lambda}, \\
\sigma_{\lambda} & :=\lambda^{\frac{N+2}{N-2}} \widetilde{\sigma} \\
\pi_{\lambda} & :=\lambda^{\frac{N+2}{N-2}} \widetilde{\pi}
\end{aligned}\right.
$$


then $\left(\phi_{\lambda}, W_{\lambda}\right)$ solves the system (2.4) with $\sigma=\sigma_{\lambda}$ and $\pi=\pi_{\lambda}$.

The proof of Theorem 3.1 follows by setting $\epsilon_{0}=\lambda_{0}^{\frac{N+2}{N-2}}$ and $\epsilon=\lambda^{\frac{N+2}{N-2}}$.

\section{AN EXISTENCE RESULT FOR $\sigma$ AND $\pi$ SMALL IN $L^{2}$}

In this section, we adapt the method of [20, 21, 29] to our context. The first step is to prove an existence result for solutions to the Lichnerowicz equation. Very nice existence results for solutions to the Lichnerowicz equation are given in [18], [31, 32] and [19]. We prove here an existence result suited to our applications. See Theorem 4.2. We then study the full system (2.4) and obtain the following theorem:

Theorem 4.1. Under the regularity assumptions stated in Section 2.2, and assuming that the operator $L_{g, \psi}$ is coercive and that $(M, g)$ has no conformal Killing vector fields, the system (2.4) admits at least one solution $(\phi, W) \in W^{2, \frac{p}{2}} \times W^{2, \frac{p}{2}}$ provided that

$$
\int\left(|\sigma|^{2}+\pi^{2}\right) d \mu^{g}
$$

is less than some small constant (depending on the seed data).

4.1. The Lichnerowicz equation. Here and in what follows, we define the following norm. Given $\phi \in H^{1}(M, g)$, we set

$$
\|\phi\|_{h}^{2}:=\int_{M}\left(\frac{4(n-1)}{n-2}|d \phi|^{2}+\mathcal{R}_{\psi} \phi^{2}\right) d \mu^{g} .
$$

Since we assumed that the modified conformal Laplacian is coercive, there exists a constant $s>0$ such that for any $\phi \in H^{1}$, we have

$$
\|\phi\|_{h}^{2} \geqslant s\|\phi\|_{L^{N}}^{2}
$$

The aim of this section is to prove the following theorem:

Theorem 4.2. Assuming that $|\sigma+\mathbb{L} W|^{2}+\pi^{2} \in L^{\frac{p}{2}}$, there exists a (small) constant $\mu=\mu\left(s,\left\|\mathcal{B}_{\tau, \psi}\right\|_{L \infty}\right)>0$ such that if

$$
0<\int_{M}\left(|\sigma+\mathbb{L} W|^{2}+\pi^{2}\right) d \mu^{g}<\mu
$$

the Lichnerowicz equation (2.4a) admits a solution $\phi \in H^{1}$ which is a stable minimizer for the functional

$$
\begin{aligned}
I_{W}(\phi):=\frac{1}{2} \int_{M} & \left(\frac{4(n-1)}{n-2}|d \phi|^{2}+\mathcal{R}_{\psi} \phi^{2}\right) d \mu^{g}-\int_{M} \frac{\mathcal{B}_{\tau, \psi}}{N} \phi^{N} d \mu^{g} \\
& +\int_{M} \frac{|\sigma+\mathbb{L} W|^{2}+\pi^{2}}{N \phi^{N}} d \mu^{g} .
\end{aligned}
$$

and whose energy satisfies

$$
\|\phi\|_{h}^{2} \leqslant C\left(\int_{M}\left(|\sigma+\mathbb{L} W|^{2}+\pi^{2}\right) d \mu^{g}\right)^{\frac{2}{N+2}} .
$$

for some constant $C=C\left(s,\left\|\mathcal{B}_{\tau, \psi}\right\|_{L^{\infty}}, \mu\right)$.

The spirit of the proof of this theorem is different from [18]. The point being that we want to obtain a stable solution $\phi_{0}$, meaning that $\phi_{0}$ is a stable local minimum for the functional $I$ defined in (4.2), while [18] uses the mountain pass lemma. Stability will ensure that the minimum $\phi_{0}$ varies continously with respect to the parameters. This will turn out to be very important when applying the Schauder fixed point theorem in Section 4.2. 
The proof of Theorem 4.2 will be carried out in the remaining of this section. For convenience, we denote

$$
A_{W}:=\int_{M}\left(|\sigma+\mathbb{L} W|^{2}+\pi^{2}\right) d \mu^{g}
$$

We also denote by $B_{R_{0}}$ the ball of radius $R_{0}>0$ centered at the origin in $H^{1}$ for the norm $\|\cdot\|_{h}$.

Lemma 4.3. There exists an $R_{0}>0$ depending only on $g$ and $\psi$ such that the functional

$$
\bar{I}(\phi):=\frac{1}{2} \int_{M}\left(\frac{4(n-1)}{n-2}|d \phi|^{2}+\mathcal{R}_{\psi} \phi^{2}\right) d \mu^{g}-\int_{M} \frac{\mathcal{B}_{\tau, \psi}}{N}|\phi|^{N} d \mu^{g}
$$

has $\operatorname{Hess} \bar{I}(\phi)(u, u) \geqslant \frac{1}{2}\|u\|_{h}^{2}$ for all $\phi \in B_{R_{0}}(0)$ and all $u \in H^{1}$.

In particular, we have

for all $\phi \in B_{R_{0}}$.

$$
\frac{1}{4}\|\phi\|_{h}^{2} \leqslant \bar{I}(\phi)
$$

Proof of Lemma 4.3. The Hessian of $\bar{I}$ at $\phi \in H^{1}$ and in the direction $u \in H^{1}$ is given by

$$
\operatorname{Hess} \bar{I}(\phi)(u, u)=\int_{M}\left[\frac{4(n-1)}{n-2}|d u|^{2}+\mathcal{R}_{\psi} u^{2}-(N-1) \mathcal{B}_{\tau, \psi}|\phi|^{N-2} u^{2}\right] d \mu^{g} .
$$

We estimate the Hessian as follows:

$$
\begin{aligned}
\operatorname{Hess} \bar{I}(\phi)(u, u) & \geqslant \int_{M}\left(\frac{4(n-1)}{n-2}|d u|^{2}+\mathcal{R}_{\psi} u^{2}\right) d \mu^{g}-(N-1)\left\|\mathcal{B}_{\tau, \psi}\right\|_{L^{\infty}}\|\phi\|_{L^{N}}^{N-2}\|u\|_{L^{N}}^{2} \\
& \geqslant\|u\|_{h}^{2}-\frac{N-1}{s}\left\|\mathcal{B}_{\tau, \psi}\right\|_{L^{\infty}}\|\phi\|_{L^{N}}^{N-2}\|u\|_{h}^{2} \\
& \geqslant\left(1-\frac{N-1}{s}\left\|\mathcal{B}_{\tau, \psi}\right\|_{L^{\infty}}\|\phi\|_{L^{N}}^{N-2}\right)\|u\|_{h}^{2}
\end{aligned}
$$

Thus, if $\|\phi\|_{L^{N}}<\left(\frac{s}{2(N-1)\left\|\mathcal{B}_{\tau, \psi}\right\|_{L^{\infty}}}\right)^{\frac{1}{N-2}}$, the Hessian of $\bar{I}$ satisfies the assumptions of the lemma. From the Sobolev embedding theorem, the conclusion of the lemma holds with

$$
R_{0}=s^{1 / 2}\left(\frac{s}{2(N-1) \mathcal{B}_{\tau, \psi}}\right)^{\frac{1}{N-2}} .
$$

We now introduce the following functional:

$$
\begin{aligned}
I_{W}^{\epsilon}(\phi):= & \frac{1}{2} \int_{M}\left(|d \phi|^{2}+\mathcal{R}_{\psi} \phi^{2}\right) d \mu^{g}-\int_{M} \frac{\mathcal{B}_{\tau, \psi}}{N}|\phi|^{N} d \mu^{g} \\
& +\int_{M} \frac{A_{W}}{N(\phi+\epsilon)^{N}} d \mu^{g}+\int_{M} \phi_{-}^{N} d \mu^{g},
\end{aligned}
$$

where $\phi_{-}:=-\min \{\phi, 0\}$. Note that the two terms we added are convex on the set

$$
\Omega_{\epsilon}:=\left\{\phi \in H^{1}, \phi \geqslant-\epsilon / 2 \text { a.e. }\right\} .
$$

This set is convex and closed for the $H^{1}$-norm. Indeed, we have

$$
\Omega_{\epsilon}=\bigcap_{f}\left\{\phi \in H^{1}, \int_{M} f \phi d \mu^{g} \geqslant-\frac{\epsilon}{2} \int_{M} f d \mu^{g}\right\}
$$

where we took the intersection over the set of (say) continuous positive functions $f$. In particular, the set $\Omega_{\epsilon}$ is compact for the weak topology on $H^{1}$. 

is

Continuity of $I_{W}^{\epsilon}$ is easy to prove. Indeed, the only difficult term to prove continuity of

$$
\int_{M} \frac{A_{W}}{N(\phi+\epsilon)^{N}} d \mu^{g}
$$

But, given $\phi_{0} \in \Omega_{\epsilon}$ and $\nu>0$, there exists $\Lambda>0$ so that

$$
\frac{1}{N}\left(\frac{2}{\epsilon}\right)^{N} \int_{A_{W} \geqslant \Lambda} A_{W} d \mu^{g} \leqslant \frac{\nu}{4}
$$

So, for any $\phi \in \Omega_{\epsilon}$, we have

$$
\begin{aligned}
\left|\int_{M} \frac{A_{W}}{N(\phi+\epsilon)^{N}} d \mu^{g}-\int_{M} \frac{A_{W}}{N\left(\phi_{0}+\epsilon\right)^{N}} d \mu^{g}\right| \\
\leqslant\left|\int_{A_{W}<\Lambda} \frac{A_{W}}{N(\phi+\epsilon)^{N}} d \mu^{g}-\int_{A_{W}<\Lambda} \frac{A_{W}}{N\left(\phi_{0}+\epsilon\right)^{N}} d \mu^{g}\right| \\
\quad+\left|\int_{A_{W} \geqslant \Lambda} \frac{A_{W}}{N(\phi+\epsilon)^{N}} d \mu^{g}\right|+\left|\int_{A_{W} \geqslant \Lambda} \frac{A_{W}}{N\left(\phi_{0}+\epsilon\right)^{N}} d \mu^{g}\right| \\
\leqslant\left|\int_{A_{W}<\Lambda}\left(\frac{A_{W}}{N(\phi+\epsilon)^{N}}-\frac{A_{W}}{N\left(\phi_{0}+\epsilon\right)^{N}}\right) d \mu^{g}\right|+\frac{\nu}{2} \\
\leqslant\left|\int_{A_{W}<\Lambda}\left(\int_{0}^{1} \frac{1}{\left(t \phi+(1-t) \phi_{0}\right)^{N+1}} d t\right)\left(\phi-\phi_{0}\right) A_{W} d \mu^{g}\right|+\frac{\nu}{2} \\
\leqslant \Lambda\left(\frac{2}{\epsilon}\right)^{-N-1}\left\|\phi-\phi_{0}\right\|_{L^{1}}+\frac{\nu}{2} .
\end{aligned}
$$

Hence, provided $\left\|\phi-\phi_{0}\right\|_{L^{1}}<\frac{\nu}{2 \Lambda}\left(\frac{2}{\epsilon}\right)^{N+1}$, we have

$$
\left|\int_{M} \frac{A_{W}}{N(\phi+\epsilon)^{N}} d \mu^{g}-\int_{M} \frac{A_{W}}{N\left(\phi_{0}+\epsilon\right)^{N}} d \mu^{g}\right|<\nu .
$$

The $H^{1}$-norm being stronger than the $L^{1}$-norm this concludes the proof of the continuity of $I_{W}^{\epsilon}$. Note that $I$ itself is continuous a priori only on $\bigcup_{\epsilon<0} \Omega_{\epsilon}$ which is not closed. This is one of the reasons why we need to regularize $I$.

Now note that since $I_{W}^{\epsilon}$ is (strictly) convex and continuous on $\Omega_{\epsilon} \cap B_{R_{0}}$ it is weakly lower semi-continuous: There exists a unique $\phi_{\epsilon} \in \Omega_{\epsilon} \cap B_{R_{0}}$ such that

$$
I_{W}^{\epsilon}\left(\phi_{\epsilon}\right)=\inf _{\phi \in \Omega_{\epsilon} \cap B_{R_{0}}} I_{W}^{\epsilon}(\phi) .
$$

The $\phi_{-}$-term in the definition of $I_{W}^{\epsilon}$ together with the strict convexity of the functional $I_{W}^{\epsilon}$ ensures that $\phi_{\epsilon} \geqslant 0$. Indeed, we see that $I_{W}^{\epsilon}(\phi) \geqslant I_{W}^{\epsilon}(|\phi|)$. It follows from elliptic regularity that $\phi_{\epsilon} \in W^{2, \frac{p}{2}}$ and from the Harnack inequality that $\phi_{\epsilon}>0$. In particular $\phi_{\epsilon} \in \Omega_{0} \cap B_{R_{0}}$.

To estimate the norm of $\phi_{\epsilon}$, we evaluate $I_{W}^{\epsilon}$ on constant functions $\phi \equiv \lambda$ :

$$
\begin{aligned}
I_{W}^{\epsilon}(\lambda) & =\frac{\lambda^{2}}{2} \int_{M} \mathcal{R}_{\psi} d \mu^{g}-\frac{\lambda^{N}}{N} \int_{M} \mathcal{B}_{\tau, \psi} d \mu^{g}+\frac{(\lambda+\epsilon)^{-N}}{N} \int_{M}\left(|\sigma+\mathbb{L} W|^{2}+\pi^{2}\right) d \mu^{g} \\
& =\frac{a}{2} \lambda^{2}-\frac{b}{N} \lambda^{N}+\frac{c}{N}(\lambda+\epsilon)^{-N},
\end{aligned}
$$

where

$$
a=\int_{M} \mathcal{R}_{\psi} d \mu^{g}, \quad b=\int_{M} \mathcal{B}_{\tau, \psi} d \mu^{g}, \quad c=\int_{M}\left(|\sigma+\mathbb{L} W|^{2}+\pi^{2}\right) d \mu^{g} .
$$


Some simple analysis shows that the stable minimum of $I_{0}(\lambda)$ is attained at some value $\lambda \sim\left(\frac{c}{a}\right)^{\frac{1}{N+2}}$. We thus have

$$
\begin{aligned}
\bar{I}\left(\phi_{\epsilon}\right) & \leqslant I_{W}^{\epsilon}\left(\phi_{\epsilon}\right) \\
& \leqslant I_{W}^{\epsilon}\left(\left(\frac{c}{a}\right)^{\frac{1}{N+2}}-\epsilon\right) \\
& \leqslant\left(\frac{1}{2}+\frac{1}{N}\right)\left(\frac{c^{2}}{a^{N}}\right)^{\frac{1}{N+2}}-\frac{b}{N}\left(\frac{c}{a}\right)^{\frac{N}{N+2}}-a \epsilon\left(\frac{c}{a}\right)^{\frac{1}{N+2}}+\frac{a}{2} \epsilon^{2} .
\end{aligned}
$$

Choosing $\epsilon \leqslant\left(\frac{c}{a}\right)^{\frac{1}{N+2}}$ and using Inequality (4.4), we get

$$
\frac{1}{4}\left\|\phi_{\epsilon}\right\|_{h}^{2} \leqslant \bar{I}\left(\phi_{\epsilon}\right) \leqslant\left(\frac{1}{2}+\frac{1}{N}\right)\left(\frac{c^{2}}{a^{N}}\right)^{\frac{1}{N+2}} .
$$

It is important to remark at this point that the estimate we got for $\left\|\phi_{\epsilon}\right\|_{h}^{2}$ is actually independent of $\epsilon$.

Following [32], we construct a (positive) sub-solution to the equation for the critical points of the functional (4.6):

$$
\frac{4(n-1)}{n-2} \Delta \phi+\mathcal{R}_{\psi} \phi=\mathcal{B}_{\tau, \psi} \phi^{N-1}+\frac{|\sigma+\mathbb{L} W|^{2}+\pi^{2}}{(\phi+\epsilon)^{N+1}}
$$

Note that the set $\Omega_{\epsilon}$ has empty interior in $H^{1}$ so one cannot speak about the Hessian of $I_{W}^{\epsilon}$ restricted to this set. Critical points are here to be understood as points for which the Gâteaux derivative of the functional $I_{W}^{\epsilon}$ vanishes in the direction of smooth functions. Nevertheless Equation (4.8) is satisfied by the function $\phi_{\epsilon}$ as long as $\left\|\phi_{\epsilon}\right\|_{h}<R_{0}$ because one then has that $\phi_{\epsilon}+t \xi \in \Omega_{\epsilon} \cap B_{R_{0}}$ for any smooth function $\xi$ as long as $|t|$ is small enough.

Since the construction of a subsolution will be useful later, we collect it in a lemma:

Lemma 4.4. There exists a positive subsolution $\phi_{\mathrm{sub}} \in W^{2, \frac{p}{2}}$ independent of $\epsilon$ to all (4.8). Further, $\phi_{\text {sub }}$ can be chosen as small as we want in $H^{1}$. If $\phi_{\mathrm{sub}}$ and $\phi_{\epsilon}$ satisfy

$$
\left\|\phi_{\epsilon}\right\|_{L^{N}},\left\|\phi_{\text {sub }}\right\|_{L^{N}}<\left(\frac{s}{(N-1)\left\|\mathcal{B}_{\tau, \psi}\right\|_{L^{\infty}}}\right)^{\frac{N}{N-2}},
$$

we have $\phi_{\epsilon} \geqslant \phi_{\text {sub }}$.

Proof. Defining $\mathcal{B}_{-}:=\min \left\{\mathcal{B}_{\tau, \psi}, 0\right\}$, and given some $\alpha$ to be chosen later, we solve the following equation for $u$ :

$$
\frac{4(n-1)}{n-2} \Delta u+\mathcal{R}_{\psi} u=|\sigma+\mathbb{L} W|^{2}+\pi^{2}+\alpha \mathcal{B}_{-} .
$$

Note that when $\alpha=0$, this equation was already studied in Step 1, Section 3. The corresponding solution $u$ was continuous and positive, hence, choosing $\alpha>0$ small enough, we still get a positive solution to (4.9).

We now set $\phi_{\text {sub }}:=\theta u$ for some $\theta>0$. As in the proof of Theorem 3.1, it can be checked that, provided $\theta$ is small enough (depending only on $\max (u)$ ), $\phi_{\text {sub }}$ is a subsolution to (4.8), namely:

$$
\frac{4(n-1)}{n-2} \Delta \phi_{\mathrm{sub}}+\mathcal{R}_{\psi} \phi_{\mathrm{sub}} \leqslant \mathcal{B}_{\tau, \psi} \phi_{\mathrm{sub}}^{N-1}+\frac{|\sigma+\mathbb{L} W|^{2}+\pi^{2}}{\left(\phi_{\mathrm{sub}}+\epsilon\right)^{N+1}} .
$$

Indeed, the condition for $\psi_{\text {sub }}$ to be a subsolution reads

$$
\left(\theta-\theta^{-N-1} u^{-N-1}\right) A_{W}+\alpha \theta \mathcal{B}_{-}-\mathcal{B} \theta^{N-1} u^{N-1} \leqslant 0,
$$

which follows from

$$
\theta\left(1-\theta^{-N-2} u^{-N-1}\right) A_{W}+\mathcal{B}_{-}\left(\alpha \theta-\theta^{N-1} u^{N-1}\right) \leqslant 0 .
$$


This last condition is fulfilled by choosing $\theta>0$ such that

$$
\left\{\begin{array} { r l } 
{ 1 } & { \leqslant \theta ^ { - N - 2 } u ^ { - N - 1 } } \\
{ \alpha \theta } & { \geqslant \theta ^ { N - 1 } u ^ { N - 1 } }
\end{array} \Leftrightarrow \left\{\begin{array}{l}
\theta \leqslant(\max u)^{\frac{N+1}{N+2}} \\
\theta \leqslant \alpha^{\frac{1}{N-2}}(\max u)^{\frac{1-N}{N-2}} .
\end{array}\right.\right.
$$

We define $\left(\phi_{\epsilon}-\phi_{\text {sub }}\right)_{-}:=\min \left\{0, \phi_{\epsilon}-\phi_{\text {sub }}\right\}$. Subtracting Equation (4.8) for $\phi_{\epsilon}$ with the previous inequality satisfied by $\phi_{\mathrm{sub}}$, multiplying by $\left(\phi_{\epsilon}-\phi_{\mathrm{sub}}\right)_{-}$and integrating over $M$, we get:

$$
\begin{aligned}
& \int_{M}\left(\frac{4(n-1)}{n-2}\left|d\left(\phi_{\epsilon}-\phi_{\mathrm{sub}}\right)_{-}\right|^{2}+\mathcal{R}_{\psi}\left(\phi_{\epsilon}-\phi_{\mathrm{sub}}\right)_{-}^{2}\right) d \mu^{g} \\
& \leqslant \int_{M} \mathcal{B}_{\tau, \psi}\left(\phi_{\epsilon}^{N-1}-\phi_{\mathrm{sub}}^{N-1}\right)\left(\phi_{\epsilon}-\phi_{\mathrm{sub}}\right)_{-} d \mu^{g} \\
& \quad+\int_{M}\left(|\sigma+\mathbb{L} W|^{2}+\pi^{2}\right)\left(\frac{1}{\left(\phi_{\epsilon}+\epsilon\right)^{N+1}}-\frac{1}{\left(\phi_{\mathrm{sub}}+\epsilon\right)^{N+1}}\right)\left(\phi_{\epsilon}-\phi_{\mathrm{sub}}\right)_{-} d \mu^{g} \\
& \leqslant(N-1) \int_{M} \mathcal{B}_{\tau, \psi}\left(\int_{0}^{1}\left(t \phi_{\epsilon}+(1-t) \phi_{\mathrm{sub}}\right)^{N-2} d t\right)\left(\phi_{\epsilon}-\phi_{\mathrm{sub}}\right)_{-}^{2} d \mu^{g} \\
& \quad-(N+1) \int_{M}\left(|\sigma+\mathbb{L} W|^{2}+\pi^{2}\right)\left(\int_{0}^{1}\left(t \phi_{\epsilon}+(1-t) \phi_{\mathrm{sub}}+\epsilon\right)^{-N-2} d t\right)\left(\phi_{\epsilon}-\phi_{\mathrm{sub}}\right)_{-}^{2} d \mu^{g} \\
& \leqslant(N-1)\left\|\mathcal{B}_{\tau, \psi}\right\|_{L^{\infty}}\left(\int_{M}\left(\int_{0}^{1}\left(t \phi_{\epsilon}+(1-t) \phi_{\mathrm{sub}}\right)^{N-2} d t\right)^{\frac{N}{N-2}} d \mu^{g}\right)^{\frac{N-2}{N}} \\
& \times\left(\int_{M}\left(\phi_{\epsilon}-\phi_{\mathrm{sub}}\right)_{-}^{N} d \mu^{g}\right)^{\frac{2}{N}}, \\
& s\left(\int_{M}\left(\phi_{\epsilon}-\phi_{\mathrm{sub}}\right)_{-}^{N} d \mu^{g}\right)^{\frac{2}{N}} \\
& \leqslant(N-1)\left\|\mathcal{B}_{\tau, \psi}\right\|_{L^{\infty}}\left(\max \left\{\left\|\phi_{\epsilon}\right\|_{L^{N}},\left\|\phi_{\mathrm{sub}}\right\|_{\left.L^{N}\right\}}\right)^{\frac{N-2}{N}}\left(\int_{M}\left(\phi_{\epsilon}-\phi_{\mathrm{sub}}\right)_{-}^{N} d \mu^{g}\right)^{\frac{2}{N}}\right.
\end{aligned}
$$

We conclude that if $(N-1)\left\|\mathcal{B}_{\tau, \psi}\right\|_{L^{\infty}}\left(\max \left\{\left\|\phi_{\epsilon}\right\|_{L^{N}},\left\|\phi_{\text {sub }}\right\|_{L^{N}}\right\}\right)^{\frac{N-2}{N}}<s,\left(\phi_{\epsilon}-\phi_{\text {sub }}\right)_{-} \equiv$ 0 which is equivalent to saying that $\phi_{\epsilon} \geqslant \phi_{\text {sub }}$.

We now let $\epsilon$ go to zero. From the fact that $\Omega_{0} \cap B_{R_{0}}$ is weakly compact, there exists $\phi_{0} \in \Omega_{0} \cap B_{R_{0}}$ which is the weak limit of some sequence $\left(\phi_{\epsilon_{i}}\right)_{i \geqslant 0}$, where $\epsilon_{i} \rightarrow 0$. We can also assume that $\phi_{\epsilon_{i}} \rightarrow \phi_{0}$ a.e..

Since all $\phi_{\epsilon}$ are greater than or equal to $\phi_{\text {sub }}$, we have $\phi_{0} \geqslant \phi_{\text {sub }}$ and

$$
\frac{|\sigma+\mathbb{L} W|^{2}+\pi^{2}}{\left(\phi_{\epsilon_{i}}+\epsilon_{i}\right)^{N+1}} \rightarrow \frac{|\sigma+\mathbb{L} W|^{2}+\pi^{2}}{\phi_{0}^{N+1}} \text { in } L^{q}
$$

for any $q<\frac{p}{2}$ since

is uniformly bounded in $L^{\infty}$ and

$$
\frac{1}{\left(\phi_{\epsilon_{i}}+\epsilon_{i}\right)^{N+1}}
$$

$$
\frac{1}{\left(\phi_{\epsilon_{i}}+\epsilon_{i}\right)^{N+1}} \rightarrow \frac{1}{\phi_{0}^{N+1}} \text { a.e.. }
$$

As a consequence $\phi_{0}$ satisfies the Lichnerowicz equation (2.4a) in a weak sense. Elliptic regularity shows that $\phi_{0} \in W^{2, \frac{p}{2}}$ and $I_{W}^{\epsilon}\left(\phi_{\epsilon}\right) \rightarrow I\left(\phi_{0}\right)$. Since $I_{W}^{\epsilon} \leqslant I$ on $B_{R_{0}} \cap \Omega_{0}$, we have

$$
I_{W}^{\epsilon}\left(\phi_{\epsilon}\right)=\min _{B_{R_{0}} \cap \Omega_{0}} I_{W}^{\epsilon} \leqslant \inf _{B_{R_{0}} \cap \Omega_{0}} I
$$


This means that $I\left(\phi_{0}\right)=\inf _{B_{R_{0}} \cap \Omega_{0}} I: \phi_{0}$ is a minimizer for $I$. From the fact that $B_{R_{0}} \cap \Omega_{0}$ is convex and $I$ is strictly convex on $B_{R_{0}} \cap \Omega_{0}$, we deduce that $\phi_{0}$ is the unique positive solution to the Lichnerowicz equation on $B_{R_{0}}$.

4.2. The coupled system. We now study the coupled system. As in [10], the operator

$$
\phi \mapsto \frac{3 n-2}{n-1} \Delta \phi+\mathcal{R}_{\psi} \phi
$$

naturally appears. We make the temporary assumption that this operator is coercive and let $s^{\prime}$ be some positive constant so that

$$
\|u\|_{k}^{2}:=\int_{M}\left(\frac{3 n-2}{n-1}|d u|^{2}+\mathcal{R}_{\psi} u^{2}\right) d \mu^{g} \geqslant s^{\prime}\|u\|_{L^{N}}^{2} .
$$

We shall even assume that $\mathcal{R}_{\psi}$ is positive in the proof. This assumption can be removed by performing a conformal change of the metric $g$, see [7, Proposition 1], and working with the conformal thin sandwich method which is explicitly conformally covariant and differs from the conformal method by the introduction of a lapse function. We refer the reader to [24] for an extensive discussion of this fact.

We are going to use a fixed point argument. Starting from $\phi_{0} \in L^{N p}$, we solve the vector equation (2.4b) with $\phi \equiv \phi_{0}$ and get $W \in W^{2, q}$, where $\frac{1}{q}=\frac{1}{p}+\frac{1}{n}$ which we plug in the Lichnerowicz equation. Assuming that $\mathbb{L} W$ is small enough in $L^{2}$, Theorem 4.2 yields a unique $\phi>0$ in $B_{R_{0}} \subset H^{1}$, which by elliptic regularity belongs to $W^{2, \frac{p}{2}} \subset L^{\infty} \subset L^{N p}$. We call this mapping (wherever it is defined) $\Phi$.

We first prove the following lemma:

Lemma 4.5. There exists $a \mu_{0}>0$ and a constant $R>0$ such that, provided

$$
\int_{M}\left(|\sigma|^{2}+\pi^{2}\right) d \mu^{g}<\mu_{0}
$$

the mapping $\Phi$ is well defined on the set

$$
C:=\left\{\phi \in L^{N p}, \int_{M} \phi^{2 N} d \mu^{g} \leqslant R\right\}
$$

and $C$ is stable for the mapping $\Phi$.

In the course of the proof, we will use the following fact: There exists a constant $\gamma>0$ such that for any $W \in H^{1}$, we have

$$
\int_{M}|\mathbb{L} W|^{2} d \mu^{g} \geqslant \gamma\left(\int_{M}|W|^{N} d \mu^{g}\right)^{2 / N}
$$

Proof. We contract the vector equation with $W$ and integrate over $M$. We obtain: 


$$
\begin{aligned}
& \frac{1}{2} \int_{M}|\mathbb{L} W|^{2} d \mu^{g}=-\frac{n-1}{n} \int_{M} \phi^{N}\langle d \tau, W\rangle+\int_{M} \pi\langle d \psi, W\rangle d \mu^{g} \\
& \leqslant \frac{n-1}{2 n}\left(\alpha \int_{M} \phi_{0}^{2 N} d \mu^{g}+\frac{1}{\alpha} \int_{M}|d \tau|^{2}|W|^{2} d \mu^{g}\right) \\
&+\frac{1}{2}\left(\beta \int_{M} \pi^{2} d \mu^{g}+\frac{1}{\beta} \int_{M}|d \psi|^{2}|W|^{2} d \mu^{g}\right) \\
& \leqslant \frac{n-1}{2 n}\left[\alpha \int_{M} \phi_{0}^{2 N} d \mu^{g}+\frac{\|d \tau\|_{L^{n}}^{2 / n}}{\alpha}\left(\int_{M}|W|^{N} d \mu^{g}\right)^{2 / N}\right] \\
&+ \frac{1}{2}\left[\beta \int_{M} \pi^{2} d \mu^{g}+\frac{\|d \psi\|_{L^{n}}^{2 / n}}{\beta}\left(\int_{M}|W|^{N} d \mu^{g}\right)^{2 / N}\right] \\
& 2 n {\left[\alpha \int_{M} \phi_{0}^{2 N} d \mu^{g}+\frac{\|d \tau\|_{L^{n}}^{2 / n}}{\alpha \gamma} \int_{M}|\mathbb{L} W|^{2} d \mu^{g}\right] } \\
&+\frac{1}{2}\left[\beta \int_{M} \pi^{2} d \mu^{g}+\frac{\|d \psi\|_{L^{n}}^{2 / n}}{\beta \gamma} \int_{M}|\mathbb{L} W|^{2} d \mu^{g}\right] \\
& \frac{n-1}{2 n} \frac{\|d \tau\|_{L^{n}}^{2 / n}}{\alpha \gamma}-\frac{1}{2} \frac{\|d \psi\|_{L^{n}}^{2 / n}}{\beta \gamma} \int_{M}|\mathbb{L} W|^{2} d \mu^{g} \\
& \frac{n-1}{2 n} \alpha \int_{M} \phi_{0}^{2 N} d \mu^{g}+\frac{1}{2} \beta \int_{M} \pi^{2} d \mu^{g} .
\end{aligned}
$$

Choosing $\alpha$ and $\beta$ large enough, we conclude that there exist constants $c_{1}$ and $c_{2}$ such that

$$
\int_{M}\left(|\sigma+\mathbb{L} W|^{2}+\pi^{2}\right) d \mu^{g} \leqslant \int_{M}|\sigma|^{2} d \mu^{g}+c_{1} \int_{M} \phi_{0}^{2 N} d \mu^{g}+\left(1+c_{2}\right) \int_{M} \pi^{2} d \mu^{g} .
$$

This proves that, if $\phi_{0} \in L^{2 N}$ and $\pi, \sigma \in L^{2}$ are small enough, $\mathbb{L} W$ is small in $L^{2}$ so Theorem 4.2 applies giving a solution $\phi$ to the Lichnerowicz equation.

Next, we multiply the Lichnerowicz equation by $\phi^{N+1}$ and integrate by parts the Laplacian:

$$
\begin{aligned}
& \frac{3 n-2}{n-1} \int_{M}\left|d \phi^{\frac{N}{2}+1}\right|^{2} d \mu^{g}+\int_{M} \mathcal{R}_{\psi} \phi^{N+2} d \mu^{g} \\
& =\int_{M} \mathcal{B}_{\tau, \psi} \phi^{2 N} d \mu^{g}+\int_{M}\left(|\sigma+\mathbb{L} W|^{2}+\pi^{2}\right) d \mu^{g} \\
& \leqslant\left\|\mathcal{B}_{\tau, \psi}\right\|_{L^{\infty}} \int_{M} \phi^{2 N} d \mu^{g}+\int_{M}\left(|\sigma|^{2}+\left(1+c_{2}\right) \pi^{2}\right) d \mu^{g}+c_{1} \int_{M} \phi_{0}^{2 N} d \mu^{g} \\
& \leqslant\left\|\mathcal{B}_{\tau, \psi}\right\|_{L^{\infty}}\left(\int_{M} \phi^{N} d \mu^{g}\right)^{\frac{N-2}{N}}\left(\int_{M} \phi^{N \frac{N+2}{2}} d \mu^{g}\right)^{\frac{2}{N}} \\
& \quad+\int_{M}\left(|\sigma|^{2}+\left(1+c_{2}\right) \pi^{2}\right) d \mu^{g}+c_{1} \int_{M} \phi_{0}^{2 N} d \mu^{g} .
\end{aligned}
$$

Hence, introducing the norm $\|\cdot\|_{k}$ (see (4.1)),

$$
\begin{aligned}
& \left(1-\frac{\left\|\mathcal{B}_{\tau, \psi}\right\|_{L^{\infty}}}{\left(s^{\prime}\right)^{1 / N}}\left(\int_{M} \phi^{N} d \mu^{g}\right)^{\frac{N-2}{N}}\right)\left\|\phi^{\frac{N}{2}+1}\right\|_{k}^{2} \\
& \leqslant \int_{M}\left(|\sigma|^{2}+\left(1+c_{2}\right) \pi^{2}\right) d \mu^{g}+c_{1} \int_{M} \phi_{0}^{2 N} d \mu^{g} .
\end{aligned}
$$


From the Sobolev embedding together with the Hölder inequality, we get:

$$
\begin{aligned}
\int_{M} \phi^{2 N} d \mu^{g} & \leqslant \operatorname{Vol}(M, g)^{\frac{N-2}{N+2}}\left\|\phi^{1+\frac{N}{2}}\right\|_{L^{N}}^{\frac{4 N}{N+2}} \\
& \leqslant \operatorname{Vol}(M, g)^{\frac{N-2}{N+2}}\left(s^{\prime}\right)^{-\frac{2 N}{N+2}}\left\|\phi^{1+\frac{N}{2}}\right\|_{k}^{\frac{4 N}{N+2}} .
\end{aligned}
$$

Thus, assuming that $\|\phi\|_{L^{N}}$ is small enough:

we conclude that

$$
\left(\int_{M} \phi^{N} d \mu^{g}\right)^{\frac{N-2}{N}} \leqslant \frac{1}{2} \frac{\left(s^{\prime}\right)^{1 / N}}{\left\|\mathcal{B}_{\tau, \psi}\right\|_{L^{\infty}}}
$$

$$
\begin{aligned}
& \frac{s^{\prime}}{2} \operatorname{Vol}(M, g)^{\frac{N-2}{2 N}}\left(\int_{M} \phi^{2 N} d \mu^{g}\right)^{\frac{N+2}{2 N}} \\
& \quad \leqslant \int_{M}\left(|\sigma|^{2}+\left(1+c_{2}\right) \pi^{2}\right) d \mu^{g}+c_{1} \int_{M} \phi_{0}^{2 N} d \mu^{g} .
\end{aligned}
$$

Denoting

$$
y=\int_{M} \phi^{2 N} d \mu^{g}, \text { resp. } y_{0}=\int_{M} \phi_{0}^{2 N} d \mu^{g},
$$

we obtain an inequality of the following form for $y$ :

$$
y \leqslant\left(\frac{x}{\lambda}+\frac{c_{1}}{\lambda} y_{0}\right)^{\frac{2 N}{N+2}},
$$

where

$$
x=\int_{M}\left(|\sigma|^{2}+\left(1+c_{2}\right) \pi^{2}\right) d \mu^{g} \text { and } \lambda=\frac{s^{\prime}}{2} \operatorname{Vol}(M, g)^{\frac{N-2}{2 N}} .
$$

We denote by $f\left(y_{0}\right)$ the right-hand side of (4.12). Note that $f$ is an increasing function. We seek for some $R>0, R \ll 1$ such that $f(R) \leqslant R$. This would have the consequence that the set

$$
C=\left\{\phi \in L^{N p}, \int_{M} \phi^{2 N} d \mu^{g} \leqslant R\right\}
$$

is stable for the mapping $\Phi$. Indeed, we would then have that, given $\phi_{0} \in C$,

$$
\int_{M} \phi^{2 N} d \mu^{g} \leqslant f\left(\int_{M} \phi_{0}^{2 N} d \mu^{g}\right) \leqslant f(C) \leqslant C .
$$

By some simple Taylor expansion, we see that $C=2\left(\frac{x}{\lambda}\right)^{\frac{2 N}{N+2}}$ does the job provided that $x$ is small enough.

The remaining steps of the proof go as in [29]. There is however a subtlety appearing here. Continuity of the solution $\phi$ of the Lichnerowicz equation (2.4a) with respect to $\mathbb{L} W$ is usually obtain by the implicit function theorem. But the set $\Omega_{0}=\left\{\phi \in H^{1}, \phi \geqslant 0\right.$ a.e. $\}$ has empty interior. Hence working on the set $C$ is not enough.

Proposition 4.6. Assuming that

$$
\int_{M}\left(|\sigma|^{2}+\pi^{2}\right) d \mu^{g}<\mu_{0}
$$

where $\mu_{0}>0$ is as defined in Lemma 4.5, there exist sequences $\left(q_{i}\right)_{i \geqslant 0}$ and $\left(R_{i}\right)_{i \geqslant 0}$, $q_{i} \geqslant 2, q_{i} \rightarrow \infty$ and $R_{i}>0$ such that, setting

$$
C_{k}:=C \cap \bigcap_{i=0}^{k}\left\{\phi \in L^{N p},\|\phi\|_{L^{N q_{i}}} \leqslant R_{i} \text { for all } i, 0 \leqslant i \leqslant k\right\},
$$

$\Phi$ maps $C_{k}$ into $C_{k+1} \subset C_{k}$. 
We use an induction argument which is quite similar in spirit to the one used in $[10,16]$. Note that, however, in these references, the Laplacian term is discarded because it vanishes for large solutions. Here it will play an important role.

Proof of Proposition 4.6. We define sequences $q_{i} \geqslant 2, R_{i}$ inductively so that

$$
\sup \left\{\|\phi\|_{L^{N q_{i}}}, \phi \in \Phi^{i}(C)\right\} \leqslant R_{i} .
$$

From Lemma 4.5, we can choose $q_{0}=2$ and $R_{0}=R^{1 / 2 N}$.

Given $\phi_{0} \in C_{i}$, we set $\phi=\Phi\left(\phi_{0}\right)$. Note that $\phi_{0} \in C_{i-1}$ (or $\phi_{0} \in C$ if $i=0$ ), hence, by induction, $\phi \in C_{i}$ (when $i=0$, this is Lemma 4.5).

The solution $W$ to the vector equation

$$
-\frac{1}{2} \mathbb{L}^{*} \mathbb{L} W=\frac{n-1}{n} \phi^{N} d \tau-\pi d \psi
$$

belongs to $W^{2, r_{i}}$, where $r_{i}$ is given by

$$
\frac{1}{r_{i}}=\frac{1}{n}+\frac{1}{q_{i}} \geqslant \frac{1}{n}
$$

By elliptic regularity, together with the Sobolev embedding,

$$
\begin{aligned}
\|\mathbb{L} W\|_{L^{q_{i}}} & \lesssim R_{i}\|d \tau\|_{L^{n}}+\|\pi d \psi\|_{L^{r_{i}}} \\
& \lesssim R_{i}+\|\pi d \psi\|_{L^{n}} .
\end{aligned}
$$

We multiply the Lichnerowicz equation for $\phi=\Phi\left(\phi_{0}\right)$ by $\phi^{N+1+2 k_{i}}$ for some $k_{i} \geqslant 0$ to be chosen later and integrate over $M$ to get:

$$
\begin{gathered}
\frac{4(n-1)}{n-2} \frac{N+1+2 k_{i}}{\left(\frac{N}{2}+1+k_{i}\right)^{2}} \int_{M}\left|d(\phi)^{\frac{N}{2}+1+k_{i}}\right|^{2} d \mu^{g}+\int_{M} \mathcal{R}_{\psi} \phi^{N+2+2 k_{i}} d \mu^{g} \\
=\int_{M} \mathcal{B}_{\tau, \psi} \phi^{2 N+2 k_{i}} d \mu^{g}+\int_{M}\left(|\sigma+\mathbb{L} W|^{2}+\pi^{2}\right) \phi^{2 k_{i}}
\end{gathered}
$$

Since we assumed that $\mathcal{R}_{\psi}>0$, there exists a constant $s_{i}>0$ so that

$\forall \xi \in H^{1}, \frac{4(n-1)}{n-2} \frac{N+1+2 k_{i}}{\left(\frac{N}{2}+1+k_{i}\right)^{2}} \int_{M}|d \xi|^{2} d \mu^{g}+\int_{M} \mathcal{R}_{\xi} \phi^{2} d \mu^{g} \geqslant s_{i}\left(\int_{M} \xi^{N} d \mu^{g}\right)^{\frac{2}{N}}$.

Applying this inequality to (4.14) with $\xi \equiv \phi^{\frac{N}{2}+1+k_{i}}$, we get:

$$
\begin{aligned}
& s_{i}\left(\int_{M} \phi^{N\left(\frac{N}{2}+1+k_{i}\right)} d \mu^{g}\right)^{2 / N} \\
& \leqslant\left\|\mathcal{B}_{\tau, \psi}\right\|_{L^{\infty}} \int_{M} \phi^{2 N+2 k_{i}} d \mu^{g}+\int_{M}\left(|\sigma+\mathbb{L} W|^{2}+\pi^{2}\right) \phi^{2 k_{i}} \\
& \leqslant\left\|\mathcal{B}_{\tau, \psi}\right\|_{L^{\infty}}\left(\int_{M} \phi^{2 N} d \mu^{g}\right)^{1-x}\left(\int_{M} \phi^{2 N+\frac{2 k_{i}}{x}} d \mu^{g}\right)^{x} \\
& \quad+\left(\int_{M}\left(|\sigma+\mathbb{L} W|^{2}+\pi^{2}\right)^{\frac{q_{i}}{2}} d \mu^{g}\right)^{\frac{2}{q_{i}}}\left(\int_{M} \phi^{2 k_{i} \frac{q_{i}}{q_{i}-2}} d \mu^{g}\right)^{1-\frac{2}{q_{i}}}
\end{aligned}
$$

where $x \in(0,1)$ is some constant to be chosen later. From Equation (4.13), we have that

$$
\left(\int_{M}\left(|\sigma+\mathbb{L} W|^{2}+\pi^{2}\right)^{\frac{q_{i}}{2}} d \mu^{g}\right)^{\frac{2}{q_{i}}} \lesssim\|\sigma\|_{L^{p}}^{2}+\|\pi\|_{L^{p}}^{2}+\|\mathbb{L} W\|_{L^{q_{i}}}^{2}
$$

is bounded from above independently of $W$ by some constant $C_{i}$. We choose $k_{i}$ so that

$$
2 k_{i} \frac{q_{i}}{q_{i}-2}=N q_{i},
$$


i.e.,

$$
k_{i}=\frac{N}{2}\left(q_{i}-2\right) \geqslant 0
$$

Note that since $\phi=\Phi\left(\phi_{0}\right) \in C$, we have that

$$
\left(\int_{M} \phi^{2 k_{i} \frac{q_{i}}{q_{i}-2}} d \mu^{g}\right)^{1-\frac{2}{q_{i}}} \leqslant R_{i}^{q_{i}-2} .
$$

We now come back to the choice of $x$. We let $x$ be such that

$$
2 N+\frac{2 k_{i}}{x}=N\left(\frac{N}{2}+1+k_{i}\right)
$$

that is to say

$$
x=\frac{2 k_{i}}{N k_{i}+N\left(\frac{N}{2}-1\right)}<\frac{2}{N} .
$$

We finally arrive at the following inequality:

$$
\begin{aligned}
& s_{i}\left(\int_{M} \phi^{N\left(\frac{N}{2}+1+k_{i}\right)} d \mu^{g}\right)^{2 / N} \\
& \quad \leqslant\left\|\mathcal{B}_{\tau, \psi}\right\|_{L^{\infty}} R^{1-x}\left(\int_{M} \phi^{N\left(\frac{N}{2}+1+k_{i}\right)} d \mu^{g}\right)^{x}+C_{i} R_{i}^{q_{i}-2} .
\end{aligned}
$$

Since $x<\frac{2}{N}$ we immediately deduce that, setting $q_{i+1}=\frac{N}{2}+1+k_{i}$,

$$
\|\phi\|_{L^{N q_{i+1}}} \leqslant R_{i+1}
$$

for some $R_{i+1}$ independent of $\phi_{0} \in C$. We have

$$
q_{i+1}=\frac{N}{2}+1+\frac{N}{2}\left(q_{i}-2\right)=\frac{N}{2}\left(q_{i}-1\right)+1
$$

so $q_{i}=1+\left(\frac{N}{2}\right)^{i}$ goes to infinity with $i$.

We point here that we were slightly sloppy. Namely for $i=0, k_{0}=0$ and $x=0$ which is not allowed in our calculation. Note however that multiplying the Lichnerowicz equation with $\phi^{2 N}$ and integrating over $M$, we get, as in the proof of Lemma 4.5, that

$$
\begin{aligned}
& \frac{3 n-2}{n-1} \int_{M}\left|d \phi^{\frac{N}{2}+1}\right|^{2} d \mu^{g}+\int_{M} \mathcal{R}_{\psi} \phi^{N+2} d \mu^{g} \\
& \quad \leqslant\left\|\mathcal{B}_{\tau, \psi}\right\|_{L^{\infty}} \int_{M} \phi^{2 N} d \mu^{g}+\int_{M}\left(|\sigma+\mathbb{L} W|^{2}+\pi^{2}\right) d \mu^{g} \\
& \quad \leqslant R\left\|\mathcal{B}_{\tau, \psi}\right\|_{L^{\infty}}+\int_{M}\left(|\sigma+\mathbb{L} W|^{2}+\pi^{2}\right) d \mu^{g}
\end{aligned}
$$

so the argument still applies.

We now choose $k$ so that $q_{k} \geqslant p$ and set $\bar{C}:=C_{k}$. We come back to the subsolution introduced in Lemma 4.4. This lemma is taken from [29].

Lemma 4.7. There exists $\eta>0$ so that all $\phi=\Phi\left(\phi_{0}\right)$ with $\phi_{0} \in \bar{C}$ satisfy $\phi \geqslant \eta$.

Proof. We study in more details the proof of Lemma 4.4. We can write $u=u_{1}-\alpha u_{2}$, where $u_{1}$ and $u_{2}$ solve

$$
\left\{\begin{array}{l}
\frac{4(n-1)}{n-2} \Delta u_{1}+\mathcal{R}_{\psi} u_{1}=|\sigma+\mathbb{L} W|^{2}+\pi^{2}, \\
\frac{4(n-1)}{n-2} \Delta u_{2}+\mathcal{R}_{\psi} u_{2}=\mathcal{B}_{+} .
\end{array}\right.
$$

The Green function $G(x, y)$ of the modified conformal Laplacian

$$
\frac{4(n-1)}{n-2} \Delta+\mathcal{R}_{\psi}
$$


is positive and continuous outside the diagonal of $M \times M$ where it blows up. Hence, there exists a constant $\epsilon>0$ such that $G(x, y) \geqslant \epsilon$. This implies that

$$
\begin{aligned}
u_{1}(x) & =\int_{M} G(x, y)\left(|\sigma+\mathbb{L} W|^{2}+\pi^{2}\right)(y) d \mu^{g}(y) \\
& \geqslant \epsilon \int_{M}\left(|\sigma+\mathbb{L} W|^{2}+\pi^{2}\right)(y) d \mu^{g}(y) \\
& \geqslant \epsilon \int_{M}\left(|\sigma|^{2}+|\mathbb{L} W|^{2}+\pi^{2}\right) d \mu^{g} \\
& \geqslant \epsilon \int_{M}\left(|\sigma|^{2}+\pi^{2}\right) d \mu^{g} .
\end{aligned}
$$

So $u_{1}$ is bounded from below independently of $W$ so $\alpha$ in the proof of Lemma 4.4 can be chosen independently of $W$ so that e.g. $u \geqslant \frac{\epsilon}{2} \int_{M}\left(|\sigma|^{2}+\pi^{2}\right) d \mu^{g}$. Since we assumed that $\phi \in \bar{C}$, we also have that

$$
|\sigma+\mathbb{L} W|^{2}+\pi^{2}
$$

is bounded from above by some constant depending on $R^{\prime}$ in $L^{p / 2}$ so $u$ is bounded in $W^{2, \frac{p}{2}} \hookrightarrow L^{\infty}$ independently of the choice of $\phi \in \bar{C}$.

Hence, the constant $\theta$ so that $\phi_{\text {sub }}=\theta u$ is a sub-solution to the Lichnerowicz equation can be chosen independently of $W$.

Setting

$$
\eta=\frac{\epsilon \theta}{2} \int_{M}\left(|\sigma|^{2}+\pi^{2}\right) d \mu^{g}
$$

we have $\phi_{\text {sub }} \geqslant \eta$ so $\phi \geqslant \eta$.

Lemma 4.8. Under the assumptions of the previous lemma, the mapping $\Phi: \bar{C} \rightarrow \bar{C}$ is continuous and compact.

Proof. We first prove continuity of the mapping $\Phi$. Assume given a sequence $\left(\phi_{i}\right)_{i}, \phi_{i} \in \bar{C}$ such that $\phi_{i} \rightarrow \phi_{\infty}$ in $L^{N p}$.

We denote with a prime their images under the mapping $\Phi: \phi_{i}^{\prime}=\Phi\left(\phi_{i}\right), \phi_{\infty}^{\prime}=\Phi\left(\phi_{\infty}\right)$. And we also denote by $W_{i}$ (resp. $W_{0}$ ) the corresponding solutions to the vector equation:

$$
\left\{\begin{aligned}
-\frac{1}{2} \mathbb{L}^{*} \mathbb{L} W_{i} & =\frac{n-1}{n} \phi_{i}^{N} d \tau-\pi d \psi, \\
-\frac{1}{2} \mathbb{L}^{*} \mathbb{L} W_{\infty} & =\frac{n-1}{n} \phi_{\infty}^{N} d \tau-\pi d \psi .
\end{aligned}\right.
$$

We have $W_{i} \rightarrow W_{\infty}$ in $W^{2, q}, \frac{1}{q}=\frac{1}{p}+\frac{1}{n}$, so $\left|\mathbb{L} W_{i}\right|^{2} \rightarrow\left|\mathbb{L} W_{\infty}\right|^{2}$ in $L^{\frac{p}{2}}$. Since the Hessian of $I_{W_{\infty}}$ is more coercive on $B_{R_{0}}$ than that of $\bar{I}$, we have from Lemma 4.3:

$$
\frac{1}{4}\left\|\phi_{i}^{\prime}-\phi_{\infty}^{\prime}\right\|_{h}^{2} \leqslant I_{W_{\infty}}\left(\phi_{i}^{\prime}\right)-I_{W_{\infty}}\left(\phi_{\infty}^{\prime}\right)
$$

for some constant $\lambda>0$. It follows from Lemma 4.7 that $\phi_{i}^{\prime} \geqslant \eta$ for all $i$ (resp. $\phi_{\infty}^{\prime} \geqslant \eta$ ). As a consequence,

$$
\begin{aligned}
\lambda\left\|\phi_{i}^{\prime}-\phi_{\infty}^{\prime}\right\|_{h}^{2} & \leqslant I_{W_{\infty}}\left(\phi_{i}^{\prime}\right)-I_{W_{\infty}}\left(\phi_{\infty}^{\prime}\right) \\
& \leqslant\left(I_{W_{\infty}}\left(\phi_{i}^{\prime}\right)-I_{W_{i}}\left(\phi_{i}^{\prime}\right)\right)+\left(I_{W_{i}}\left(\phi_{i}^{\prime}\right)-I_{W_{\infty}}\left(\phi_{\infty}^{\prime}\right)\right) \\
& \leqslant \frac{\eta^{-N}}{N}\left\|\left|\mathbb{L} W_{i}\right|^{2}-\left|\mathbb{L} W_{\infty}\right|^{2}\right\|_{L^{1}}+\sup _{\phi \in B_{R_{0}} \cap \Omega_{-2 \eta}}\left|I_{W_{i}}(\phi)-I_{W_{\infty}}(\phi)\right| \\
& \leqslant 2 \frac{\eta^{-N}}{N}\left\|\left|\mathbb{L} W_{i}\right|^{2}-\left|\mathbb{L} W_{\infty}\right|^{2}\right\|_{L^{1}},
\end{aligned}
$$

where to pass from the second line to the third, we used the fact that the map "infimum" is 1-Lipschitzian. Thus we get that $\phi_{i}^{\prime} \rightarrow \phi_{\infty}^{\prime}$ in $H^{1}$ and in particular $\phi_{i}^{\prime} \rightarrow \phi_{\infty}^{\prime}$ in $L^{N}$. 
Convergence in $L^{N p}$ follows from elliptic regularity. Indeed, looking at the Lichnerowicz equation for $\phi_{i}^{\prime}$ :

$$
\frac{4(n-1)}{n-2} \Delta \phi_{i}^{\prime}+\mathcal{R}_{\psi} \phi_{i}^{\prime}=\mathcal{B}_{\tau, \psi}\left(\phi_{i}^{\prime}\right)^{N-1}+\frac{\left|\sigma+\mathbb{L} W_{i}\right|^{2}+\pi^{2}}{\left(\phi_{i}^{\prime}\right)^{N+1}},
$$

we see that the righthand side is bounded in $L^{\frac{p}{2}}$ independently of $i$, as a consequence of Lemma 4.7. So the sequence $\left(\phi_{i}^{\prime}\right)$ is bounded in $W^{2, \frac{p}{2}} \hookrightarrow L^{\infty}$. By interpolation, $\left(\phi_{i}^{\prime}\right)$ is a Cauchy sequence in $L^{N p}$ whose limit in $L^{N}$ is $\phi_{\infty}^{\prime}$. We conclude that $\phi_{i}^{\prime} \rightarrow \phi_{\infty}^{\prime}$ in $L^{N p}$.

Compactness of the mapping $\Phi$ is fairly simple since we noticed that the set $(\Phi(C))^{\frac{N+2}{2}}$ is bounded in $H^{1}$ (this is Estimate (4.11)) so $\Phi(C)$ embeds compactly in $L^{2 N}$ by the Rellich theorem. Then notice that pursuing one step further the proof of Proposition 4.6, the set $\Phi(\bar{C})$ is bounded in $L^{N q_{K+1}}$. Compactness of $\Phi(\bar{C})$ for the $L^{N p}$-norm follows by interpolation.

Theorem 4.1 then follows by applying the Schauder fixed point theorem. Namely, the convex hull of $\Phi(\bar{C}) \subset \bar{C}$ is compact, convex and stable for the mapping $\Phi$. So $\Phi$ admits a fixed point $\phi \in \bar{C}$ which is in turn a solution to the conformal constraint equations.

\section{REFERENCES}

[1] R. Bartnik and J. Isenberg, The constraint equations, The Einstein equations and the large scale behavior of gravitational fields, Birkhäuser, Basel, 2004, pp. 1-38. 1, 2, 3

[2] R. Beig, P.T. Chruściel, and R. Schoen, KIDs are non-generic, Ann. Henri Poincaré 6 (2005), no. 1, 155194. 4

[3] Y. Choquet-Bruhat, General relativity and the Einstein equations, Oxford Mathematical Monographs, Oxford University Press, Oxford, 2009. 3

[4] Y. Choquet-Bruhat and R. Geroch, Global aspects of the Cauchy problem in general relativity, Comm. Math. Phys. 14 (1969), 329-335. 3

[5] Y. Choquet-Bruhat, J. Isenberg, and D. Pollack, The Einstein-scalar field constraints on asymptotically Euclidean manifolds, Chinese Ann. Math. Ser. B 27 (2006), no. 1, 31-52. 2

[6] Y. Choquet-Bruhat, J. Isenberg, and D. Pollack, Applications of theorems of Jean Leray to the Einsteinscalar field equations, J. Fixed Point Theory Appl. 1 (2007), no. 1, 31-46. (2007k:83014) 3

[7] The constraint equations for the Einstein-scalar field system on compact manifolds, Classical Quantum Gravity 24 (2007), no. 4, 809-828. 2, 3, 12

[8] P. Chruściel and R. Gicquaud, Bifurcating solutions of the Lichnerowicz equation, submitted. 2

[9] J. Corvino and R. M. Schoen, On the asymptotics for the vacuum Einstein constraint equations, J. Differential Geom. 73 (2006), no. 2, 185-217. 2

[10] M. Dahl, R. Gicquaud, and E. Humbert, A limit equation associated to the solvability of the vacuum Einstein constraint equations by using the conformal method, Duke Math. J. 161 (2012), no. 14, 2669-2697. 2, 6, 12,15

[11] J. Dilts, J. Isenberg, R. Mazzeo, and C. Meier, Non-CMC solutions of the Einstein constraint equations on asymptotically Euclidean manifolds, Class. Quantum Grav. 31 (2014), no. 6, 065001, 10. 2

[12] J. Dilts and J. Leach, A limit equation criterion for applying the conformal method to asymptotically cylindrical initial data sets, arXiv:1401.5369. 2

[13] Y. Fourès-Bruhat, Théorème d'existence pour certains systèmes d'équations aux dérivées partielles non linéaires, Acta Math. 88 (1952), 141-225. 3

[14] R. Gicquaud and C. Huneau, Limit equation for vacuum Einstein constraint with a translational Killing vector field in the compact hyperbolic case, Submitted, arXiv:1409.3477. 6

[15] R. Gicquaud and Q.A. Ngô, A new point of view on the solutions to the Einstein constraint equations with arbitrary mean curvature and small TT-tensor, Class. Quantum Grav. 31 (2014), no. 19, 195014 (20pp). 1, 2, 4, 5

[16] R. Gicquaud and A. Sakovich, A large class of non-constant mean curvature solutions of the Einstein constraint equations on an asymptotically hyperbolic manifold, Comm. Math. Phys. 310 (2012), no. 3, 705-763. 2,15

[17] D. Gilbarg and N.S. Trudinger, Elliptic partial differential equations of second order, Classics in Mathematics, Springer-Verlag, Berlin, 2001, Reprint of the 1998 edition. 5

[18] E. Hebey, F. Pacard, and D. Pollack, A variational analysis of Einstein-scalar field Lichnerowicz equations on compact Riemannian manifolds, Comm. Math. Phys. 278 (2008), no. 1, 117-132. 2, 7

[19] E .Hebey and G. Veronelli, The Lichnerowicz equation in the closed case of the Einstein-Maxwell theory, Trans. Amer. Math. Soc. 366 (2014), no. 3, 1179-1193. 7 
[20] M. Holst, G. Nagy, and G. Tsogtgerel, Far-from-constant mean curvature solutions of Einstein's constraint equations with positive Yamabe metrics, Phys. Rev. Lett. 100 (2008), no. 16, 161101, 4. 1, 2, 4, 7

[21] _ Rough solutions of the Einstein constraints on closed manifolds without near-CMC conditions, Comm. Math. Phys. 288 (2009), no. 2, 547-613. 1, 2, 4, 7

[22] J. Isenberg, Constant mean curvature solutions of the Einstein constraint equations on closed manifolds, Class. Quantum Grav. 12 (1995), no. 9, 2249-2274. 2

[23] J.M. Lee and T.H. Parker, The Yamabe problem, Bull. Amer. Math. Soc. (N.S.) 17 (1987), no. 1, 37-91. 2

[24] D. Maxwell, The conformal method and the conformal thin-sandwich method are the same, arXiv:1402.5585. 2, 12

[25] _ Conformal parameterizations of slices of flat Kasner spacetimes, arXiv:1404.7242. 2

[26] _ Initial data in general relativity described by expansion, conformal deformation and drift, arXiv:1407.1467. 2

[27] Rough solutions of the Einstein constraint equations on compact manifolds, J. Hyperbolic Differ. Equ. 2 (2005), no. 2, 521-546. 6

[28] Solutions of the Einstein constraint equations with apparent horizon boundaries, Comm. Math. Phys. 253 (2005), no. 3, 561-583. 2

[29] A class of solutions of the vacuum Einstein constraint equations with freely specified mean curvature, Math. Res. Lett. 16 (2009), no. 4, 627-645. 1, 2, 4, 5, 7, 14, 16

[30] T. C. Nguyen, Applications of fixed point theorems to the vacuum Einstein constraint equations with nonconstant mean curvature, arXiv:1405.7731. 2

[31] B. Premoselli, Effective multiplicity for the Einstein-scalar field Lichnerowicz equation, Calc. Var. Partial Differential Equations (2014), 1-36. 2, 7

[32] _ The Einstein-scalar field constraint system in the positive case, Comm. Math. Phys. 326 (2014), no. 2, 543-557. 2, 7, 10

[33] H. Ringström, The Cauchy problem in general relativity, ESI Lectures in Mathematics and Physics, European Mathematical Society (EMS), Zürich, 2009. 3

[34] A. Sakovich, Constant mean curvature solutions of the Einstein-scalar field constraint equations on asymptotically hyperbolic manifolds, Class. Quantum Grav. 27 (2010), no. 24, 12 pages. 2

(R. Gicquaud) Laboratoire de MATHÉmatiques ET DE Physique ThÉORIQUe, Université FrançOIS RABElais de TOURs, PARC DE GRANDMOnt, 37200 TOURs, FRANCE

E-mail address: romain.gicquaudelmpt.univ-tours.fr

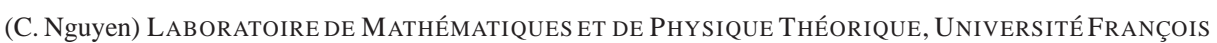

RABElais de Tours, PARC DE GRANDMOnt, 37200 Tours, FRANCE

E-mail address: The-Cang.Nguyenelmpt . univ-tours.fr 